\title{
Catestatin (Cst) protects the brain against ischemia/reperfusion injury through suppressing ER-stress and mitochondrial dysfunction
}

Pei Bing ( $\square$ yanghua2016@sohu.com )

suanqian frist people's hospital https://orcid.org/0000-0003-4641-7373

Chunjie Song

suqian frist people's hospital

Zhengjiang Zhang

suqian frist people's hospital

Shen Xin

suqian frist people's hospital

Cui Qian

suqian frsit people's hospital

\section{Research}

Keywords: Cerebral ischemia/reperfusion, Catestatin, ER-stress, mitochondrial

Posted Date: August 14th, 2020

DOI: https://doi.org/10.21203/rs.3.rs-58676/v1

License: (c) (1) This work is licensed under a Creative Commons Attribution 4.0 International License. Read Full License 


\section{Abstract}

\section{Background}

Cerebral stroke, known as a cerebral vascular accident (CVA), is one of the leading causes of long-term disability and the second leading cause of death worldwide. Despite amounts of advances that have been achieved in terms of the treatment of ischemic stroke. But thus far, clinically effective neuroprotectants remain elusive, which may mainly due to the lack of a complete understanding of molecular mechanisms of the stroke. Previous studies have been revealed that catestatin (Cst) is closely related to cardiovascular ischemia/reperfusion injuries. However, little is known about whether Cst is involved in the regulation of neuronal death processes during ischemia.

Methods

In the present study, we revealed a protective function of Cst on Rat neuron cell death in the setting of ischemia/reperfusion injury.

Results

We found that Cst treatment significantly attenuated the deficits of hippocampal related behaviors. On mechanism, our data revealed that Cst administration remarkably reduced ER-stress and mitochondrial dysfunction caused by I/R injury, and subsequently protected brain cells from apoptosis.

Conclusion

In sum, our results demonstrate that Cst ameliorates I/R injury-induced hippocampal-related behaviors deficits by protecting the neurons from I/R injury-induced ER-stress and mitochondrial dysfunction and apoptosis. Our findings may provide a promising novel neuroprotectant for ischemic stroke therapy.

\section{Background}

Cerebral stroke is also known as a cerebral vascular accident (CVA), which is one of the leading causes of long-term disability and ranks as the second leading cause of death worldwide[1-4]. The prevalence is expected to increase further as a result of population aging and increasing, an ongoing high prevalence of risk factors, and inadequate management $[3,5,6]$. It is projected that the number of death from stroke could rise to 7.8 million in 2030 [2,3]. Despite improved access to stroke treatment and overall health services, the outcome remains unsatisfactory, which may mainly because of the lack of a complete understanding of the pathophysiological processes of the stroke. Thus, further investigates in the pathologic and molecular mechanisms related to stroke is compelling needed.

Among all types of stroke, the incidence of ischemic stroke is accounting for $60 \% \sim 70 \%$ that is suspected as the most common cause of stroke. The pathophysiological mechanisms of stroke are complex, however, ischemia/reperfusion (I/R) injury is well accepted to be the main cause of cerebral 
stroke $[2,4]$. I/R injury could cause neuronal cell death by multiple distinct signaling pathways, including the induction of reactive oxygen species (ROS), mitochondrial dysfunction and endoplasmic reticulum stress (ER-stress) [1, 4, 7]. Hippocampus is a cortical structure for the brain that controls behaviors [8-15], and is most sensitive to ischemia. Thus, ischemic stroke-induced hippocampus neuron death and then behaviors injury is a major cause of stroke-related long-term disability. Various kinds of mechanisms are involved in hippocampi neuronal cell death, such as necrosis, apoptosis, and autophagy [1, 7, 16-21]. Among these mechanisms, accumulated pieces of evidence have demonstrated that apoptosis is the key event for brain damage in cerebral ischemia [4], and mitochondrial apoptosis was reported to be fundamental for I/R injury-induced hippocampi neuronal cell death and cerebral dysfunction [22-26]. Due to the high energy demand, the nerve cells are more susceptible to mitochondrial dysfunction, which may result in the burst of ROS production and the weakened mitochondrial membrane [26], then cytochrome $c$ release and neuronal apoptosis [23, 24]. Besides, the endoplasmic reticulum (ER) stress is also found to be involved in I/R injury-induced neuron apoptosis [20,27-29]. In the context of cerebral ischemia, the accumulation of unfolded protein in ER was induced and then causes an ER stress response [26], via multiple mechanisms, including activation of PERK $[1,18,19,26,30]$.

Amounts of advances have been achieved in terms of the treatment of ischemic stroke. But thus far, clinically effective neuroprotectants remain elusive. Peptide catestatin (Cst, bCHGA344-364) is a 21 amino acid-residue that derives from chromogranin-A (CHGA ) cleavage [31-34]. CST was firstly identified as a potent nicotinic-cholinergic antagonist [35], that shows characteristic inhibitory effects on nicotinic cationic $(\mathrm{Na}+, \mathrm{Ca} 2+)$ signal transduction specific to the neuronal nicotinic receptor[35]. CST is wellestablished to act as a potent inhibitor of nicotine-evoked catecholamine secretion in vitro and in vivo and represents a novel, autocrine homeostatic mechanism controlling catecholamine release from chromaffin cells and noradrenergic neurons[35]. In mammals, Cst is observed mainly expressed and secreted in granule cells and adrenergic neurons [36], and reported to be involved in the regulation of many natural and pathological physiological processes [37-39]. Moreover, CST is reported to play crucial roles in the neuronal system [36], and contained an anti-hypertensive effect in the cardiovascular system. However, the function and mechanism of Cst on cerebral ischemia/reperfusion (I/R) injury remains unknown.

In this study, we revealed a protective function of Cst on Rat neuron cell death in the context of I/Rinduced injury in vivo. Our results showed that the deficits of hippocampal related behaviors were remarkably reduced in the administration with Cst. Furthermore, our data revealed that Cst administration dramatically inhibited ER-stress and mitochondrial dysfunction caused by I/R injury, and subsequently protected brain cells from apoptosis. In sum, our results demonstrate that Cst ameliorates I/R injury-induced hippocampal-related behavior deficits by protecting the neurons from I/R injury-induced ER-stress and mitochondrial dysfunction. Our findings may provide a promising novel neuroprotectant for ischemic stroke therapy.

\section{Materials And Methods}




\section{Materials}

The human wild-type CST, hChgA352-372(SSMKLSFRARGYGFRGPGPQL), was purchased from Phoenix Pharmaceuticals (Belmont, CA, USA). Anti-Cytochrome C (SAB4502234) antibody was obtained from Sigma-Aldrich. Anti- Caspase-9 (9502), anti- cleaved-Caspase-9 (9505), anti-p-PERK (3179), anti-IRE1 (3294), Anti-ATF4 (D4B8)(\#11815), Anti-CHOP (L63F7) (\#2895), and anti- PERK (5683) were commercially obtained from Cell Signaling Biotechnology. Anti-IRE1 (phospho S724) (ab48187), and AntiGADD34 antibody (ab126075) were purchased from Abcam, and anti-b-actin(sc-10731) were obtained from Santa Cruz Biotechnology.

\section{Animals, ischemic/reperfusion model, and Cst administration}

All animal procedures complied with the Animal Management Rule. The care and use of the laboratory animals were approved by the Laboratory Animal Ethics Committee of Suqian First People's Hospital. Experiments were performed on adult male Sprague-Dawley (SD) rats (180-200 g, 8 weeks), supplied by Jining Medical University, raised at a constant temperature. The scientific project was supervised and approved by Suqian First People's Hospital.

The schedule of Cst treatment and the behavioral test was graphically presented as in Fig 1A. All rats were randomly divided into four groups: control group, I/R group, I/R and Cst-treatment group, I/R, and vehicle-treatment group. Each group contained 6 independent animals.

The surgical procedures were described previously[18]. In brief, after being anesthetized, transient cerebral ischemia was induced by four-vessel occlusion (4-VO) before rats' both vertebral arteries were occluded permanently by electrocautery and common carotid arteries were exposed, subsequently, rats were recovered for $24 \mathrm{~h}$ and fasted overnight. To induce cerebral ischemia, aneurysm clips were used to occlude both carotid arteries. After 15 min of the occlusion, the aneurysm clips were removed for reperfusion.

The administration of Cst into the rat was described previously and with slight modulation[40]. In brief, rats were started to be administered with catestatin 2 days after adaptation to the experimental environment by intraperitoneal injection. Considering the half-life of catestatin (12 hours), its administration was done daily for 7 days $(0.25 \mathrm{mg} / \mathrm{kg}$ per day B.W).

\section{Open field test}

The open field apparatus consisted of a square, opaque acrylic container, and a video camera fixed $1 \mathrm{~m}$ above the arena for tracking the rats' movement. A computerized tracking system was used to analyze the images and measure the speed and distance of movement. The individual rat was placed in the 
middle of the chamber for each trial. After adaptation, the behavior of each rat was recorded. During the interval between trials, rats were returned to its home cage in the same room and the open field was wiped clean with a slightly damp cloth. The number of rearing events, grooming sessions, total distance, and speed traveled were recorded. Meanwhile, to assess the anxiety of rats in the open field, the time spent in the central area was recorded. All rats were randomly divided into four groups: control group, I/R group, I/R and Cst-treatment group, I/R, and vehicle-treatment group. Each group contained 6 independent animals.

\section{Morris water maze (MWM) testing}

MWM testing was employed to evaluate the memory and learning capacity on the $7^{\text {th }}$ day after reperfusion and ischemia. In brief, the rats were given 3 swimming trials per day and lasted for 4 consecutive days. The rat was given a maximum of $120 \mathrm{~s}$ to find the hidden platform and allowed to stay on it for $20 \mathrm{~s}$. The proportion of time and swimming distance that the rat spent in the quadrant where the platform previously located was recorded and used as a measure of memory. Morris maze performance was analyzed for latency and distance using the ANY-maze video tracking system (Stoelting, Wood Dale, IL, USA) with a CCD camera. Each group contained 6 independent animals.

\section{Histological analysis}

Rats were anesthetized with chloral hydrate and underwent perfusion with PBS, followed by $4 \%$ paraformaldehyde in $0.1 \mathrm{M}$ phosphate buffer $(\mathrm{PB})$. Brains were removed, post-fixed overnight in paraformaldehyde, processed, and embedded in paraffin. Coronal brain sections were cut and deparaffinized in xylene and rehydrated in a gradient of ethanol and distilled water. The sections were stained using hematoxylin and eosin and examined with a light microscope. The neuronal density of the hippocampal CA1 pyramidal cells represented the number of cells per $1 \mathrm{~mm}$ length which was counted under a light microscope. Each group contained 6 independent animals, and 12 slides from per rat were randomly selected for statistic analysis.

\section{Immunofluorescence and TUNEL assay}

Tissue sections were deparaffinized in xylene and rehydrated in a gradient of ethanol and distilled water and then washed in PBS, permeabilized in $0.25 \%$ PBS-Triton X-100 and blocked with $3 \%$ BSA for 30 min. Then, the slices were incubated overnight at $4{ }^{\circ} \mathrm{C}$ with the primary antibody. Following PBS washes, secondary antibodies were incubated at room temperature for $1 \mathrm{~h}$. Slices were mounted on Fluroshield with DAPI and coverslipped. TUNEL assay was performed following the manufacturer's instruction for a kit (\#G3250, Promega, Madison, WI, USA). Slides were examined using a confocal laser-scanning microscope (Flouview FV 1000, Olympus, Japan), and the photos were analyzed using Image J software. 
Each group contained 6 independent animals, and 6 sections from per rat were randomly selected for statistic analysis.

\section{Western blot}

The concentration of proteins from tissue homogenates or cell lysates were quantified using Easy II Protein Quantitative Kit (based on BCA, Transgen Biotech). Equal protein samples were electrophoresed using SDS-PAGE gel and then were transferred to the PVDF membrane. After blocking using $5 \%(\mathrm{w} / \mathrm{v})$ BSA in TBST for $60 \mathrm{~min}$ at room temperature, primary antibodies were incubated in recommended dilution at $4^{\circ} \mathrm{C}$ overnight, and the HRP-conjugated secondary antibodies were incubated. Proteins were detected using Tanon ${ }^{\text {TM }}$ High-sig ECL Western Blotting Substrate Kit (Tanon, CAT: 180-501) according to recommended instructions. The protein bands were scanned and analyzed using Bio-Rad ChemiDoc MP (Bio-Rad CAT: 170-8280).

\section{RNA Extraction and Real-Time Quantitative Polymerase Chain Reaction (RT-qPCR)}

Total RNA was extracted from rats hippocampus neurons by Qiagen RNeasy Mini Kit according to the manufacturer's instruction. Equal amount RNA was subjected to reverse transcription for cDNAs using Takara PrimeScript RT-PCR Kit (Takara, Dalian, China).

Real-time PCR was carried out using the TB Green Fast qPCR Mix (Takara, Dalian, China). Quantification was done using ${ }^{\triangle \triangle} \mathrm{Ct}$ values and reference gene GAPDH was used as the internal control. The primers used in the present study were listed as follows: ATF4 forward, 5'-ATG GCG CTC TTC ACG AAA TC-3'; ATF4 reverse: 5'-ACT GGT CGA AGG GGT CAT CAA-3'; CHOP forward: 5'- GTC CTG TCC TCA GAT GAA ATT GG-3'; CHOP reverse: 5'- GCA GGG TCA AGA GTA GTG AAG GTT-3'; GADD34 forward: 5'- TTT CTA GGC CAG ACA CAT GG-3'; GADD34 reverse: 5'- TGT TCC TTT TTC CTC CGT GG-3'; GAPDH forward: 5'-ATC ACT GCC ACC CAG AAG AC-3'; GAPDH reverse: 5'-ATG AGG TCC ACC ACC CTG TT-3'.

\section{Measurement of Reactive Oxygen Species(ROS) Production}

The intracellular reactive oxygen species (ROS) was determined using 2,7-dichlorodihydrofluorescein diacetate (H2-DCFDA), an intracellular ROS indicator, as previously reported[26]. Cultured cortical neurons were incubated with $10 \mathrm{nM} \mathrm{H2-DCFDA}$ for 1 hat 37_C in a dark place. Then, the neurons were resuspended with PBS before being read by a fluorescence plate reader (excitation wavelength of $480 \mathrm{~nm}$, the emission wavelength of $530 \mathrm{~nm}$ ).

\section{Measurement of Mitochondrial Membrane Potential (MMP)}


To monitor the mitochondrial membrane potential (MMP), the neurons were incubated in $10 \mathrm{mM}$ fluorescent dye rhodamine 123 (RH 123), and subsequently, washed with PBS three times before reading the fluorescence with a fluorescence plate reader (excitation wavelength of $480 \mathrm{~nm}$, the emission wavelength of $530 \mathrm{~nm})[26]$.

\section{Statistical evaluation}

For animal experiments, six animals were independently selected as samples in all groups for behavior tests, western blotting, and histology assays. Image $\mathrm{J}$ software was used for semi-quantitative analysis of the bands. Values were expressed as the means \pm SEM. Student's $t$-test was used for the mean comparison between two groups, and two-way ANOVA was used for multiple group comparisons followed by Bonferron correction post hoc test. $p$-value $<0.05$ were considered to be statistically significant.

\section{Results}

\section{Cst ameliorates the deficits of hippocampal-related behaviors caused by ischemia}

We assessed the effects of Cst treatment on neuron-protection in vivo in the context of ischemic/reperfusion by the open field test (Fig 1A). As illustrated in Fig 1B-C, the value of distance moved was observed significantly decreased in I/R group compared to the control group. On the contrary, the moving distance was remarkably restored treated with Cst, compared to the vehicle control (Fig 1B, 1C). Similarly, the reduction in rats' movement average speed was detected in I/R and I/R plus vehicle treatment, and this decrease was significantly restored by Cst treatment (Fig 1C). Collectively, these results above indicated that Cst significantly ameliorated the deficit in locomotion caused by ischemia.

Variable rearing numbers represented exploratory behavior, and the grooming number and time in the corner could be considered as indexes of anxiety[12]. Consistent with locomotion, dramatically decreased values of rearing number and grooming number and time in the corner were also observed in the $I / R$ group, and the addition of Cst significantly improved all these values (Fig. 1D-F). Taken together, these data strongly suggested that Cst administration ameliorates the impairments in the locomotive and exploratory behaviors as well as anxiety modulation caused by I/R injury in rats.

\section{Cst improved the learning and spatial memory ability of Cerebral Ischemic-Reperfusion rats}

To further evaluate the protective effects of Cst on I/R injury rats, we conducted the morris water maze (MWM) tests in the setting of I/R injury [11]. We observed that the latency of I/R rats was significantly 
longer than that of control rats by day 4, day 5, and day 6 (Fig. 2A). Cst treatment notably shortened the latency in the I/R group (Fig. 2A), while vehicle treatment exerted no effect. Consistently, after ischemia, the proportion of time and the number of crossing decreased (Fig. 2B-D), on the 7th day, whereas, the I/R plus Cst group significantly improved the learning and spatial memory ability of cerebral I/R rats (Fig. $2 B-D$ ). Collectively, our data strongly indicated that Cst remarkably improved the learning and spatial memory ability of the rats in the setting of $\mathrm{I} / \mathrm{R}$ induced brain dysfunction.

\section{Cst increases neuron surviving in ischemic hippocampus}

Large cell death could occur in the ischemic hippocampus, which is thought to be the main cause of dysfunction related behaviors[41]. H\&E staining was usually applied to examine the survival rate of neurons (Fig. 3A). As illustrated by Fig.3B-C, most neurons showed normal and typical nuclear shape in the control hippocampus indicating the surviving cells, but in the I/R group, the proportion of surviving neurons shown dramatic decreased (Fig. 3B, C). Intriguingly, the number of the surviving neuron was significantly increased after Cst treatment (Fig. 3 B, C). Consistently, our TUNEL staining also showed that $\mathrm{I} / \mathrm{R}$ injury dramatically induced neurons apoptosis while CST treatment significantly protected neurons from death (Fig. 3 D, E). We further explored the effects of Cst administration on neurons surviving in the ischemic hippocampus by western blot analysis of caspas3 cleavage. As shown in Fig 3F, the caspase3 cleavage induced by I/R injury was reduced by CST treatment. Taking together, these data suggested that the Cst administration significantly reduced I/R-induced neuron cell death in the ischemic hippocampus.

\section{Cst reduces cytochrome c cytoplasmic release and endoplasmic reticulum (ER) stress in neurons which underwent I/R injury}

Our data above strongly suggested that Cst treatment significantly ameliorated the hippocampal-related behaviors dysfunction caused by $\mathrm{I} / \mathrm{R}$ induced brain injury through protecting from hippocampus neurons death. Accumulated research has demonstrated that $I / R$ induced neurons cell death mainly results from the ER-stress and cytochrome c cytoplasmic release induced mitochondrial apoptosis[22, 24, 25, 42]. It was well established that dysfunction of mitochondria plays a key role in the pathogenesis of many nervous system diseases such as stroke and neurodegenerative diseases[22, 24]. After transient focal ischemia, cytochrome $\mathrm{c}$ was been released from mitochondria to the cytosol, which could contribute acutely to further mitochondrial dysfunction and to promote neuronal death[21, 23, 26, 27]. To explore the regulatory effects of Cst on mitochondria dysfunction in hippocampus neurons underwent I/R injury, cytochrome c cytoplasmic release was determined by western blot. We found that the ration of cytoplasmic/mitochondrial Cytochrome $\mathrm{C}$ in $\mathrm{l} / \mathrm{R}$ group was up-regulated to about 3-fold compared to control (FIG. 4A, 4B), whereas Cst treatment dramatic inhibited the cytoplasmic expression of cytochrome $\mathrm{C}$ and the ration of cytoplasmic/mitochondrial cytochrome $\mathrm{C}$ in I/R neurons (FIG. 4A, 4B), indicating that 
Cst treatment can significantly improve hippocampus neurons mitochondria dysfunction caused by ischemic injury.

Furthermore, we examined the effects of CST on the expression of ER-stress markers ATF4, GADD34, and $\mathrm{CHOP}$, however, there was no influence of I/R injury or CST treatment on the expression of these markers on mRNA or protein level (FIG. 5A, 5B). It is well established that I/R injury can induce ER-stress by assessing phosphorylated PERK and IRE1[18, 26, 43]. Thus, we determined the endoplasmic reticulum stress markers $\mathrm{p}$-PERK and $\mathrm{p}$-IRE1 by immunoblot. Rats hippocampus neurons were collected and lysed to exact protein, and then the supernatants were subject to immunoblot to analyze the ER-stress markers PERK and IRE. Our results showed that p-PERK and p-IRE1 were strongly up-regulated in I/R group compared to the control group, whereas significantly down-regulated after Cst treatment (FIG. 5C-E), suggested that I/R induced hippocampus neurons ER-stress while Cst treatment remarkably rescued this phenotype. Consistently, immunofluorescence staining also illustrated a dramatic increase in p-PERK expression in I/R group, compared to the control, but significantly reduced in I/R plus Cst treatment on rats hippocampus slices (FIG. 5F-G). These data indicated that Cst significantly protects hippocampus neurons from death by suppressing mitochondria dysfunction and ER-stress induced by ischemic.

\section{Cst blocks MMP dysregulation and ROS accumulation induced activation of caspase- 9 induced by I/R in hippocampus neurons}

Studies have found that ER-stress and mitochondrial dysfunction significantly contributed to cerebral ischemia injury-induced acute neuronal cell death $[7,26]$. Mitochondrial released cytochrome $C$ correspondingly activates caspase-9 clevage which then induces cell apoptosis[7, 21, 23, 27]. Our data above revealed that Cst inhibited ER-stress and mitochondria dysfunction induced by ischemic injury in Rat hippocampus. To explore if Cst influences caspase-9 activation in hippocampus neurons underwent I/R injury, we employed western blot to check the cleaved caspase-9. Results revealed that the cleaved caspase- 9 was clearly increased after I/R in neurons but significantly reduced while Cst administration (FIG. 6A, 6B). Mitochondria dysfunction also leads to the burst of ROS production and hinders oxidative phosphorylation as the mitochondrial membrane breaks down and MMP loss[26]. We thus evaluated the MMP loss and ROS production, and results showed that I/R injury-induced neurons MMP loss and ROS production whereas Cst treatment dramatically rescued these phenotypes (FIG. 6C, 6D). Collectively, these data strongly indicated that Cst administration suppressed I/R injury-induced neurons apoptosis through abrogating MMP dysregulation and ROS accumulation induced activation of caspase-9.

\section{Discussion}

Cerebral stroke is one of the leading causes of long-term disability and the second leading cause of death worldwide[2, 3]. Despite amounts of advances that have been achieved in terms of the treatment of ischemic stroke[3, 4], thus far, clinically effective neuroprotectants remain elusive, which may mainly due 
to the lack of a complete understanding of molecular mechanisms of the stroke. Ischemic stroke leads to hippocampus related behavior dysfunction and disability, which mainly resulted from the neuron cell death induced by I/R injury [1]. Thus, the recovery of behavior dysfunction could be of great benefit for the ischemic stroke patient. In the present study, we discovered for the first time that Cst administration significantly ameliorates impairments of locomotion, exploratory activity, anxiety modulation, and cognition in the I/R rat model, which strongly suggested that Cst may be provided as a promising neuroprotectant for stroke treatment.

Neuronal cell death is an important event related to ischemic stroke. I/R induced neuronal damage in the hippocampus can cause disorders of behaviors $[1,4,7]$, deficits of locomotive activity, as well as dysfunction of anxiety mediation[1, 4, 7, 25], and ultimately results in disability or death. On mechanism, I/R injury lead to the dysfunction of mitochondrial by ROS production, and the dis-regulation of ER stress. Then, these impairments together result in neuron apoptosis. Peptide Cst is a 21 amino acid-residue, which derives from chromogranin-A cleavage [31-33]. CST involved in the regulation of many natural and pathological physiological processes, for example, in inflammation [37-39]. Importantly, CST is reported to play crucial roles in the neuronal system [36], and contained an anti-hypertensive effect in the cardiovascular system. Cst has a cardioprotective role mainly due to its functions on the direct reduction of post-ischemic myocardial damages and dysfunction [44, 45]. Our results suggested a protective effect of Cst on the hippocampus that underwent I/R injury. Our findings revealed that the treatment of Cst improved the survival of the hippocampal neuron in the I/R brain by significantly improving endoplasmic reticulum stress and mitochondria dysfunction and significantly reverse the impairments of cytochrome $c$ cytoplasmic release, MMP loss and ROS production caused by I/R injury. On mechanism, we observed that IR injury significantly activated ER stress as evidenced by the hyperphosphorylation of PERK and IRE1 and enhanced fluorescence intensity of p-PERK, which was restored by CST administration. Our western blot results showed less phosphorylation of the IRE1 and PERK proteins and reduced fluorescence intensity of phosphorylated PERK in the Cst treatment group, indicating that Cst reduces I/R injury-induced ER-stress. Taken together, our findings provide novel perceptions about the relationship between Cst, ER stress, and mitochondrial dysfunction in neurons under I/R conditions.

In conclusion, our findings demonstrated that Cst significantly ameliorates impairments of locomotion, exploratory activity, anxiety modulation, and cognition in the I/R rat model. On mechanism, Cst may protect against I/R neuronal injury by preserving mitochondrial function and suppressing ER stress.

\section{Declarations}

\section{Ethics approval and consent to participate}

All animal procedures complied with the Animal Management Rule. The care and use of the laboratory animals were approved by the Laboratory Animal Ethics Committee of Suqian First People's Hospital. 


\section{Consent for publication}

No

\section{Availability of data and material}

The datasets used and/or analysed during the current study are available from the corresponding author on request

\section{Competing interests}

The authors declare no competing financial interests.

\section{Funding}

This work was supported by the Jiangsu province natural science foundation of China (BK20161211)

\section{Authors' contributions}

Study design: PB. Experiments and data collection: CS, ZZ, SX, CQ. Data analysis and interpretation: CS. Manuscript writing: PB.

\section{Acknowledgements}

Suqian First People's Hospital

\section{References}

1. Enzmann, G., S. Kargaran, and B. Engelhardt, (2018).Ischemia-reperfusion injury in stroke: impact of the brain barriers and brain immune privilege on neutrophil function. Therapeutic advances in neurological disorders. 11: p. 1756286418794184-1756286418794184.

2. Johnston, S.C., S. Mendis, and C.D. Mathers, (2009). Global variation in stroke burden and mortality: estimates from monitoring, surveillance, and modelling. Lancet Neurology. 8(4): p. 345-354.

3. Kathleen, S., M. Colin, and B. Ruth, (2007).Preventing stroke: saving lives around the world. Lancet Neurology. 6(2): p. 182-187.

4. Nour, M., F. Scalzo, and D.S. Liebeskind, (2013).Ischemia-Reperfusion Injury in Stroke. Interventional Neurology. 1(3-4): p. 185. 
5. Johnston, S.C., S. Mendis, and C.D. Mathers, (2009). Global variation in stroke burden and mortality: estimates from monitoring, surveillance, and modelling. Lancet Neurol. 8(4): p. 345-54.

6. Strong, K., C. Mathers, and R. Bonita, (2007). Preventing stroke: saving lives around the world. Lancet Neurol. 6(2): p. 182-7.

7. White, B.C., J.M. Sullivan, D.J. Degracia, B.J. O’Neil, R.W. Neumar, L.I. Grossman, J.A. Rafols, and G.S. Krause, (2000). Brain ischemia and reperfusion: molecular mechanisms of neuronal injury. Journal of the Neurological Sciences. 179(1): p. 1-33.

8. Douglas, R.J., (1967). The hippocampus and behavior. Psychological Bulletin. 67(6): p. 416.

9. Ellen, P., (1980). Cognitive maps and the hippocampus. Physiological Psychology. 8(2): p. 168-174.

10. López Ruiz, J.R., L.P. Osuna Carrasco, C.L. López Valenzuela, N.E. Franco Rodríguez, I.T.V.B. De, E.I. Jiménez, J.M. Dueñas Jiménez, and S.H. Dueñas Jiménez, (2015). The hippocampus participates in the control of locomotion speed. Neuroscience. 311: p. 207-215.

11. Mizumori, S.J., P.A. Garcia, M.A. Raja, and B.T. Volpe, (1995).Spatial- and locomotion-related neural representation in rat hippocampus following long-term survival from ischemia. Behavioral Neuroscience. 109(6): p. 1081.

12. Nigel, F. and S. Robin, (1987). Relationships between the superior colliculus and hippocampus: Neural and behavioral considerations. Behavioral \& Brain Sciences. 10(1): p. 19.

13. Samsdodd, F., B.K. Lipska, and D.R. Weinberger, (1997).Neonatal lesions of the rat ventral hippocampus result in hyperlocomotion and deficits in social behaviour in adulthood. Psychopharmacology. 132(3): p. 303-310.

14. Takahashi, H., Y. Takada, N. Nagai, T. Urano, and A. Takada, (2000).Serotonergic neurons projecting to hippocampus activate locomotion. Brain Research. 869(1): p. 194-202.

15. Zhang, W., Q. Zhang, W. Deng, Y. Li, G. Xing, X. Shi, and Y. Du, (2014). Neuroprotective effect of pretreatment with ganoderma lucidum in cerebral ischemia/reperfusion injury in rat hippocampus. Neural regeneration research. 9(15): p. 1446-1452.

16. Chen, C.H., Z. Jiang, J.H. Yan, L. Yang, K. Wang, Y.Y. Chen, J.Y. Han, J.H. Zhang, and C.M. Zhou, (2013). The Involvement of Programmed Cell Death 5 (PDCD5) in the Regulation of Apoptosis in Cerebral Ischemia/Reperfusion Injury. Cns Neuroscience \& Therapeutics. 19(8): p. 566-576.

17. Chouchani, Edward T., Victoria R. Pell, Andrew M. James, Lorraine M. Work, K. Saeb-Parsy, C. Frezza, T. Krieg, and Michael P. Murphy, (2016).A Unifying Mechanism for Mitochondrial Superoxide Production during Ischemia-Reperfusion Injury. Cell Metabolism. 23(2): p. 254-263.

18. Feng, D., B. Wang, L. Wang, N. Abraham, K. Tao, L. Huang, W. Shi, Y. Dong, and Y. Qu, (2017).Preischemia melatonin treatment alleviated acute neuronal injury after ischemic stroke by inhibiting $E R$ stress-dependent autophagy via PERK and IRE1 signalings. Journal of Pineal Research. 62(3): $\mathrm{p}$. e12395.

19. Gong, L., Y. Tang, R. An, M. Lin, L. Chen, and J. Du, (2017).RTN1-C mediates cerebral ischemia/reperfusion injury via ER stress and mitochondria-associated apoptosis pathways. Cell Death \&Amp; Disease. 8: p. e3080. 
20. Zhang, H., W. Yan, A. Zhu, D. Huang, S. Deng, J. Cheng, M.X. Zhu, and L. Yong, (2016).SUMO-specific protease 1 protects neurons from apoptotic death during transient brain ischemia/reperfusion. Cell Death \& Disease. 7(11): p. e2484.

21. Zhang, X., H. Yan, Y. Yuan, J. Gao, Z. Shen, Y. Cheng, Y. Shen, R.R. Wang, X. Wang, and W.W. Hu, (2013).Cerebral ischemia-reperfusion-induced autophagy protects against neuronal injury by mitochondrial clearance. Autophagy. 9(9): p. 1321-1333.

22. Fiskum, G., Mitochondrial Dysfunction in the Pathogenesis of Acute Neural Cell Death. 2002.

23. Fujimura, M., Y. Morita-Fujimura, K. Murakami, M. Kawase, and P.H. Chan, (1998).Cytosolic Redistribution of Cytochrome $C$ after Transient Focal Cerebral Ischemia in Rats. Journal of Cerebral Blood Flow \& Metabolism. 18(11): p. 1239-1247.

24. Pérez-Pinzón, M.A., G.P. Xu, J. Born, J. Lorenzo, R. Busto, M. Rosenthal, and T.J. Sick, (1999). Cytochrome $C$ Is Released from Mitochondria Into the Cytosol after Cerebral Anoxia or Ischemia. Journal of Cerebral Blood Flow \& Metabolism. 19(1): p. 39-43.

25. Plesnila, N., (2004). Role of mitochondrial proteins for neuronal cell death after focal cerebral ischemia. Acta Neurochirurgica Supplement. 89(89): p. 15.

26. Wei, J., X. Wu, P. Luo, K. Yue, Y. Yu, J. Pu, L. Zhang, S. Dai, D. Han, and Z. Fei, (2019).Homer1a Attenuates Endoplasmic Reticulum Stress-Induced Mitochondrial Stress After Ischemic Reperfusion Injury by Inhibiting the PERK Pathway. Frontiers in cellular neuroscience. 13: p. 101-101.

27. Joza, N., S.A. Susin, E. Daugas, W.L. Stanford, S.K. Cho, C.Y. Li, T. Sasaki, A.J. Elia, H.Y. Cheng, and L. Ravagnan, (2001).Essential role of the mitochondrial apoptosis-inducing factor in programmed cell death. Nature. 410(6828): p. 549-554.

28. Lopez, J. and S.W. Tait, (2015).Mitochondrial apoptosis: killing cancer using the enemy within. British Journal of Cancer. 112(6): p. 957-962.

29. Williams, G.T. and C.A. Smith, (1993).Molecular regulation of apoptosis: Genetic controls on cell death. Cell. 74(5): p. 777-779.

30. Andrienko, T.N., P. Pasdois, G.C. Pereira, M.J. Ovens, and A.P. Halestrap, (2017). The role of succinate and ROS in reperfusion injury - A critical appraisal. Journal of Molecular and Cellular Cardiology. 110: p. 1-14.

31. Bandyopadhyay, G.K., C.U. Vu, S. Gentile, H. Lee, N. Biswas, N.W. Chi, D.T. O'Connor, and S.K. Mahata, (2012). Catestatin (chromogranin A(352-372)) and novel effects on mobilization of fat from adipose tissue through regulation of adrenergic and leptin signaling. Journal of Biological Chemistry. 287(27): p. 23141.

32. Bassino, E., S. Fornero, M.P. Gallo, C. Gallina, S. Femminò, R. Levi, B. Tota, and G. Alloatti, (2015).Catestatin Exerts Direct Protective Effects on Rat Cardiomyocytes Undergoing Ischemia/Reperfusion by Stimulating PI3K-Akt-GSK3 $\beta$ Pathway and Preserving Mitochondrial Membrane Potential. Plos One. 10(3): p. e0119790.

33. Wu, Z. and D. Zhu, (2014). The important role of catestatin in cardiac remodeling. Biomarkers. 19(8): p. 625-630. 
34. Mahata, S.K., M. Kiranmayi, and N.R. Mahapatra, (2018).Catestatin: A Master Regulator of Cardiovascular Functions. Curr Med Chem. 25(11): p. 1352-1374.

35. Mahata, S.K., M. Mahata, M.M. Fung, and D.T. O'Connor, (2010).Catestatin: a multifunctional peptide from chromogranin A. Regul Pept. 162(1-3): p. 33-43.

36. Claudia, P., A. Giuseppe, G. Maria Pia, C. Maria Carmela, L. Renzo, T. Francesca, B. Eleonora, D. Serena, S.K. Mahata, and T. Bruno, (2010). Catestatin improves post-ischemic left ventricular function and decreases ischemia/reperfusion injury in heart. Cellular \& Molecular Neurobiology. 30(8): p. 1171-1179.

37. Helle, K.B. and A. Corti, (2015).Chromogranin A: a paradoxical player in angiogenesis and vascular biology. Cellular and Molecular Life Sciences. 72(2): p. 339-348.

38. Mahata, S.K., M. Mahata, M.M. Fung, and D.T. O'Connor, (2010).Catestatin: a multifunctional peptide from chromogranin A. Regulatory Peptides. 162(1): p. 33-43.

39. Muntjewerff, E.M., G. Dunkel, M.J.T. Nicolasen, S.K. Mahata, and G. van den Bogaart, (2018). Catestatin as a Target for Treatment of Inflammatory Diseases. Frontiers in Immunology. 9.

40. Wang, D., T. Liu, S. Shi, R. Li, Y. Shan, Y. Huang, D. Hu, and C. Huang, (2016).Chronic Administration of Catestatin Improves Autonomic Function and Exerts Cardioprotective Effects in Myocardial Infarction Rats. J Cardiovasc Pharmacol Ther. 21(6): p. 526-535.

41. Deshpande, J., K. Bergstedt, T. Lindén, H. Kalimo, and T. Wieloch, (1992).Ultrastructural changes in the hippocampal CA1 region following transient cerebral ischemia: evidence against programmed cell death. Experimental Brain Research. 88(1): p. 91-105.

42. Kalpage, H.A., V. Bazylianska, M.A. Recanati, A. Fite, J. Liu, J. Wan, N. Mantena, M.H. Malek, I. Podgorski, E.I. Heath, A. Vaishnav, B.F. Edwards, L.I. Grossman, T.H. Sanderson, I. Lee, and M. Hüttemann, (2018). Tissue-specific regulation of cytochrome $c$ by post-translational modifications: respiration, the mitochondrial membrane potential, ROS, and apoptosis. The FASEB Journal. 33(2): p. 1540-1553.

43. Kumar, R., ., S. Azam, ., J.M. Sullivan, C. Owen, ., D.R. Cavener, P. Zhang, ., D. Ron, ., H.P. Harding, J.J. Chen, and A. Han, . (2010). Brain ischemia and reperfusion activates the eukaryotic initiation factor 2alpha kinase, PERK. Journal of Neurochemistry. 77(5): p. 1418-1421.

44. Maria-Giulia, P., T. Francesca, A. Carmelina, C. Maria Carmela, A. Tommaso, T. Bruno, A. Giuseppe, P. Claudia, and P. Pasquale, (2013). Catestatin reduces myocardial ischaemia/reperfusion injury: involvement of PI3K/AKt, PKCs, mitochondrial KATP channels and ROS signalling. Pflügers Archiv European Journal of Physiology. 465(7): p. 1031-1040.

45. Penna, C., G. Alloatti, M.P. Gallo, M.C. Cerra, R. Levi, F. Tullio, E. Bassino, S. Dolgetta, S.K. Mahata, B. Tota, and P. Pagliaro, (2010). Catestatin improves post-ischemic left ventricular function and decreases ischemia/reperfusion injury in heart. Cellular and molecular neurobiology. 30(8): p. 11711179. 


\section{Figures}

A

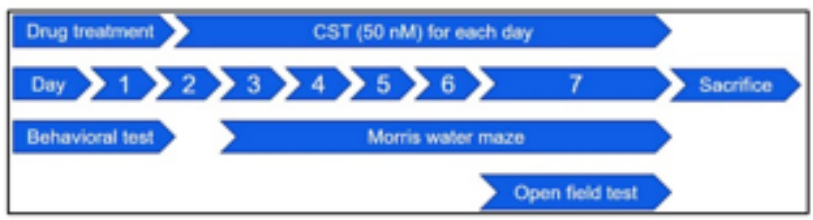

B

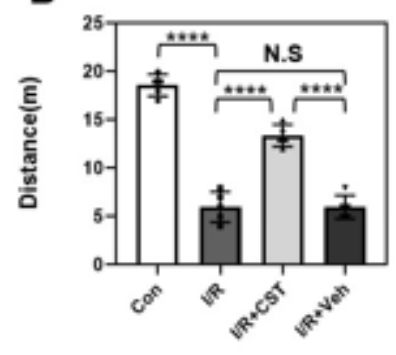

E

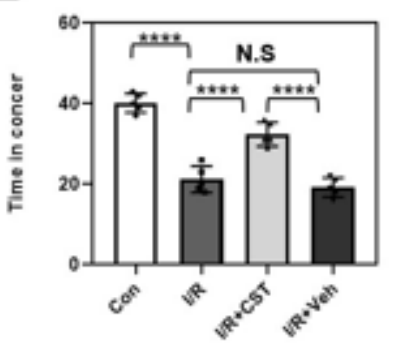

C

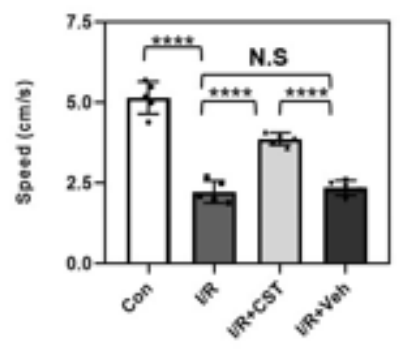

$\mathbf{F}$

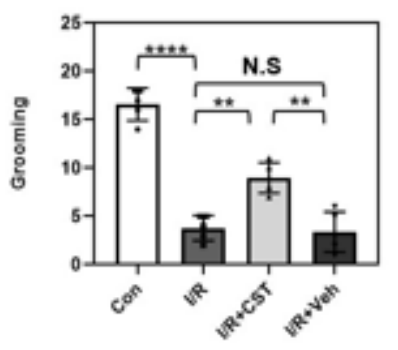

D

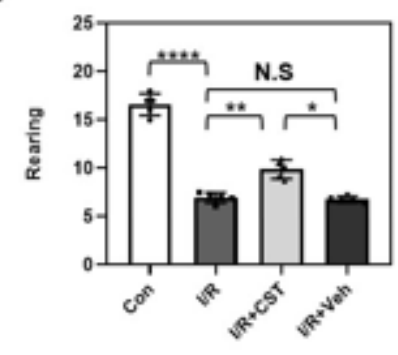

\section{Figure 1}

Cst ameliorates the deficits of hippocampal-related behaviors caused by ischemia A. The schedule of drug treatments and behavioral measurements. B. Behaviors in the open field test. Rats were divided into control group (Con), I/R group (I/R), I/R plus Cst group (I/R+CST), I/R plus vehicle group (I/R+Veh). Effect on the moving distance, $n=6$ each group, ${ }^{\star \star \star \star}$, means $p$-value $<0.001$, N.S means no significance. $C$. Effect of Cst on speed in each group. The results were expressed as the mean $\pm S D ; n=5$; $\star \star \star \star$, means $p$ value $<0.001$, N.S means no significance. D. Effect of Cst on rearing in each group. The results were expressed as the mean \pm Cst ameliorates the deficits of hippocampal-related behaviors caused by ischemia A. The schedule of drug treatments and behavioral measurements. B. Behaviors in the open field test. Rats were divided into control group (Con), l/R group (I/R), I/R plus Cst group (I/R+CST), I/R plus vehicle group ( $(1 / R+V e h)$. Effect on the moving distance, $n=6$ each group, ${ }^{\star} * \star *$, means $p$-value < 0.001 , N.S means no significance. C. Effect of Cst on speed in each group. The results were expressed as the mean $\pm S D ; n=5 ; * \star \star \star$, means $p$-value $<0.001$, N.S means no significance. D. Effect of Cst on rearing in each group. The results were expressed as the mean $\pm S D ; n=5$; $* \star \star \star$, means $p$-value $<0.001, * \star, p$ - 
value $<0.01$, *, p-value $<0.05$, N.S means no significance. E. Effect of Cst on time in corner in each group. The results were expressed as the mean $\pm S D ; n=5$; $\star \star \star \star$, means $p$-value $<0.001$, N.S means no significance. F. Effect of Cst on grooming in each group. The results were expressed as the mean \pm SD; $n$ $=5 ; * \star \star \star$, means $p$-value $<0.001, * \star, p$-value $<0.01$, N.S means no significance.

A

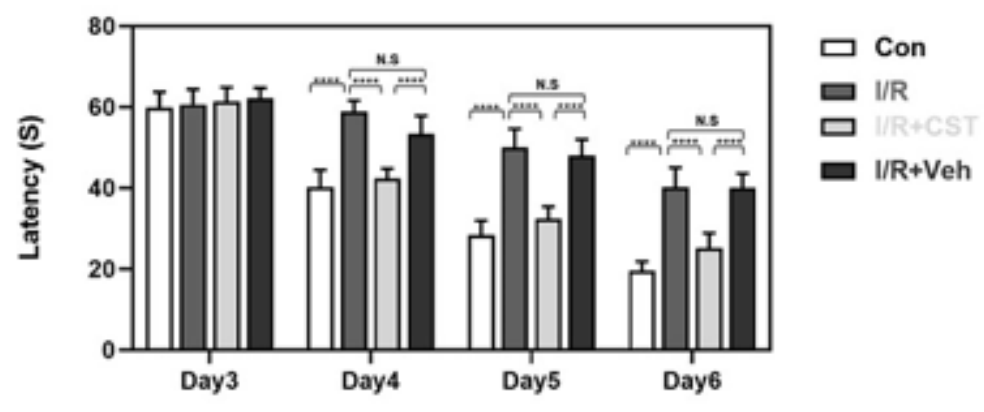

B

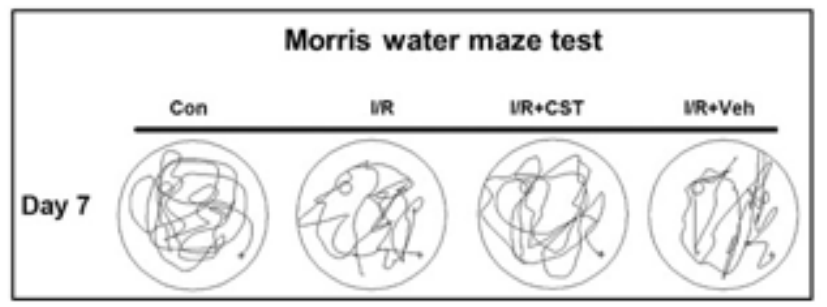

C

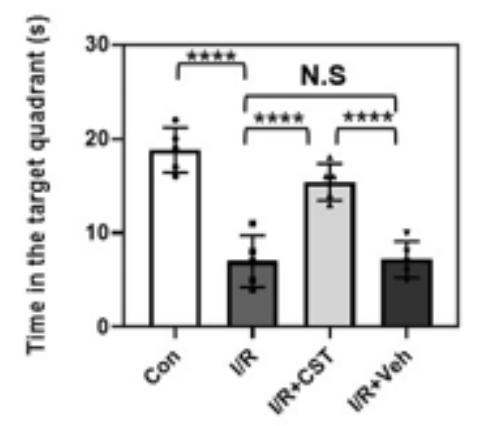

D

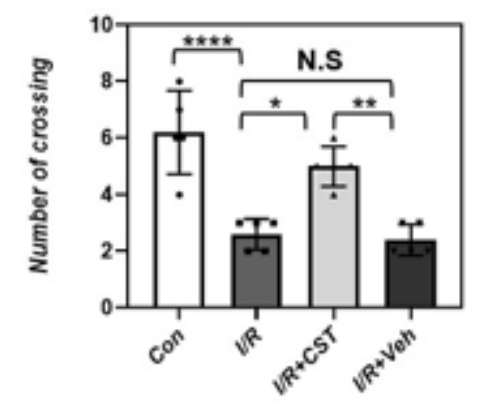

\section{Figure 2}

Cst ameliorates the deficits of hippocampal-related behaviors caused by ischemia $A$. The schedule of drug treatments and behavioral measurements. B. Behaviors in the open field test. Rats were divided into control group (Con), I/R group (I/R), I/R plus Cst group (I/R+CST), I/R plus vehicle group (I/R+Veh). Effect on the moving distance, $n=6$ each group, ${ }^{\star \star \star *}$, means $p$-value $<0.001$, N.S means no significance. $C$. Effect of Cst on speed in each group. The results were expressed as the mean $\pm S D ; n=5$; $* \star \star \star$, means $p$ value $<0.001$, N.S means no significance. D. Effect of Cst on rearing in each group. The results were expressed as the mean $\pm S D ; n=5 ; * \star \star *$, means $p$-value $<0.001$, $* *$, $p$-value $<0.01$, $*$, $p$-value $<0.05, N . S$ means no significance. E. Effect of Cst on time in corner in each group. The results were expressed as the 
mean $\pm S D ; n=5 ; * \star \star \star$, means $p$-value $<0.001$, N.S means no significance. F. Effect of Cst on grooming in each group. The results were expressed as the mean $\pm S D ; n=5 ; \star \star \star \star$, means $p$-value $<0.001, * \star, p$-value $<$ 0.01 , N.S means no significance.

A

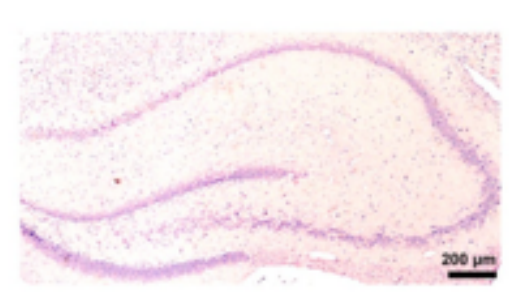

D

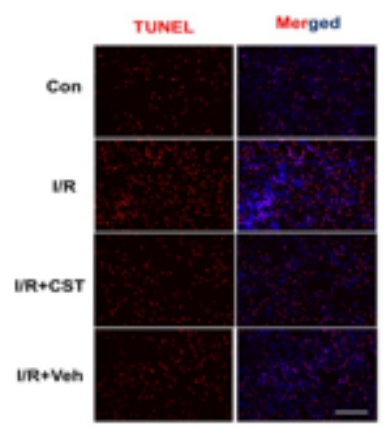

B

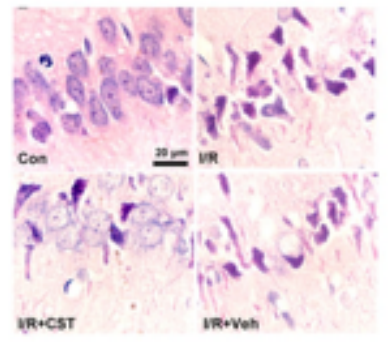

E

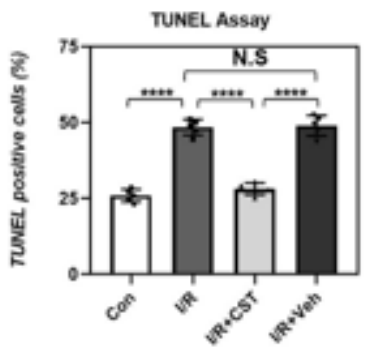

C

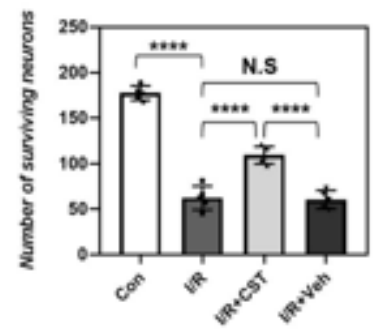

$\mathbf{F}$

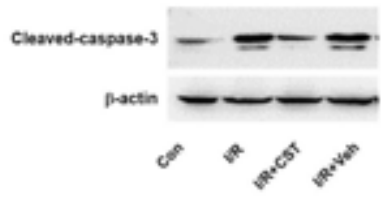

Figure 3

Effects of CST treatment on the Rats neuronal survival A. H\&E staining was performed on brain sections in each group. Picture representative hippocampal image of H\&E staining. $B$. The picture shows H\&E staining of hippocampal neurons in each group, control group (Con), l/R group (I/R), l/R plus Cst group $(\mathrm{I} / \mathrm{R}+\mathrm{CST}), \mathrm{I} / \mathrm{R}$ plus vehicle group $(\mathrm{I} / \mathrm{R}+\mathrm{Veh})$. $\mathrm{C}$. The survival neurons were counted and the number was considered as neuron quantity. Data were presented as mean $\pm S D$. $N=5$, $\star \star \star \star$, means $p$-value $<0.001, N$.S means no significance. D. The picture shows TUNEL staining of hippocampal neurons in each group, control group (Con), I/R group (I/R), I/R plus Cst group (I/R+CST), I/R plus vehicle group (I/R+Veh). E. TUNEL positive neurons were counted in five random fields and the ratio of TUNEL staining cells was calculated. Data were presented as mean $\pm S D$. $N=5$, $* \star \star \star$, means $p$-value $<0.001, N$.S means no significance. F. Western blot showed caspase3 cleavage in control (Con), I/R group (I/R), I/R plus Cst group (I/R+CST), $1 / R$ plus vehicle $(I / R+V$ eh) groups. 
A

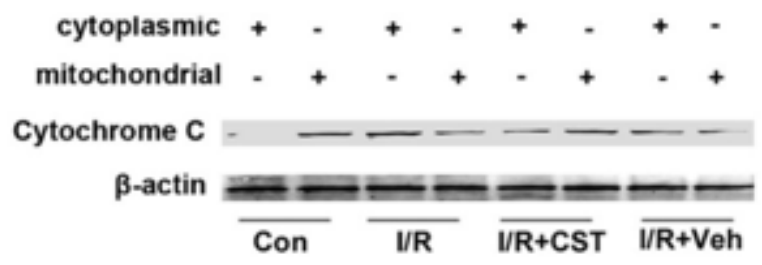

B

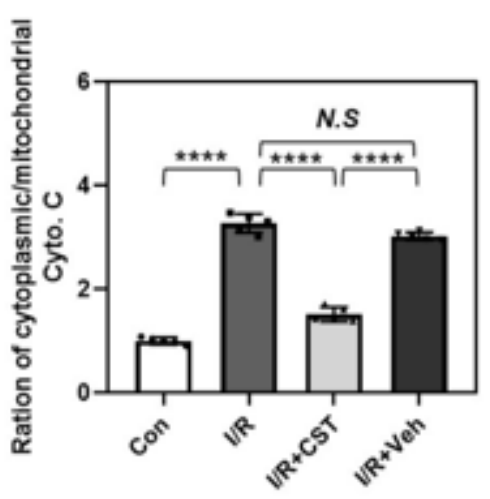

Figure 4

Effects of Cst administration on cytochrome c release in ischemic hippocampus A. Effects of Cst on cytochrome c cytoplasmic release in ischemia hippocampus neurons. Western blot results of cytoplasmic and mitochondrial cytochrome $c$ were shown, control group (Con), l/R group (I/R), l/R plus Cst group $(\mathrm{I} / \mathrm{R}+\mathrm{CST}), \mathrm{I} / \mathrm{R}$ plus vehicle group (I/R+Veh). $\mathrm{B}$. Quantitative analysis of cytochrome $c$ cytoplasmic release in each group, control: Con, I/R: I/R, I/R plus Cst: I/R+CST, I/R plus vehicle: I/R+Veh, Data were presented as mean $\pm S D$. $N=5$, $* \star \star \star$, means $p$-value $<0.001$, N.S means no significance. 
A

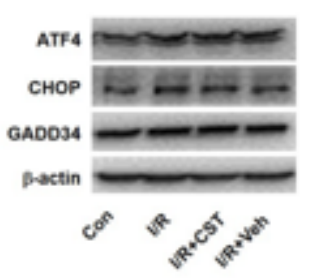

C

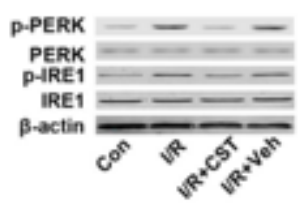

$\mathbf{F}$

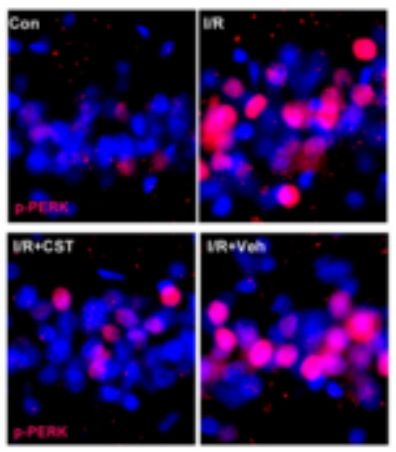

B

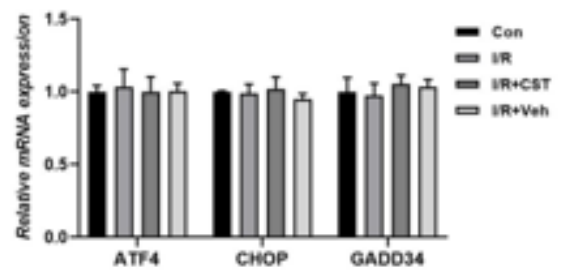

D

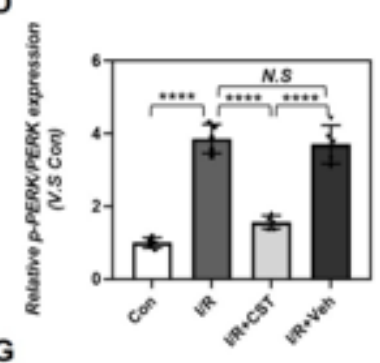

E

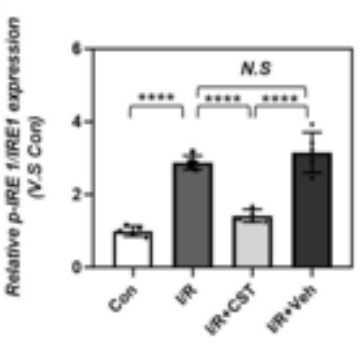

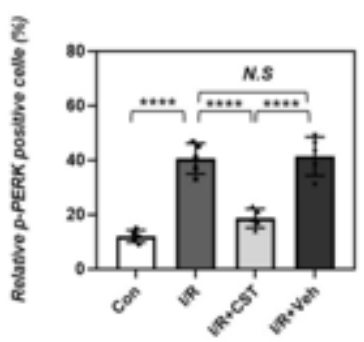

Figure 5

Effects of Cst administration on endoplasmic reticulum stress in ischemic hippocampus A. Western blot showed the protein expression of ATF4, CHOP, and GADD34 in ischemic hippocampus, control group (Con), I/R group (I/R), I/R plus Cst group (I/R+CST), I/R plus vehicle group (I/R+Veh). B. Quantitative RTPCR analysis of the mRNA expression of ATF4, CHOP, and GADD34 in the ischemic hippocampus. Data were presented as mean $\pm S D$. $N=3$, N.S means no significance. C. Western blot results of PERK and IRE1 phosphorylation in each group, Con: control, I/R: ischemia/reperfusion, Cst: catestatin, Veh: vehicle. D. Quantification of p-PERK/PERK expression in each group. The bands' intensity was expressed as optical density analysis. Data were given as mean $\pm S D$. $N=5$, $* \star \star \star$, means $p$-value $<0.001$, N.S means no significance. Con: control, I/R: ischemia/reperfusion, Cst: catestatin, Veh: vehicle. E. Quantification of pIRE1/IRE1 expression in each group. The bands' intensity was expressed as optical density analysis. Data were given as mean $\pm S D$. $N=5$, $\star \star \star \star$, means $p$-value $<0.001$, N.S means no significance. Con: control, I/R: ischemia/reperfusion, Cst: catestatin, Veh: vehicle. F. Immunofluorescence staining of p-PERK on Rats brain slices in each group, Con: control, I/R: ischemia/reperfusion, Cst: catestatin, Veh: vehicle. G. Quantification of immunofluorescence staining of p-PERK on Rats brain slices in each group, p-PERK positive neurons were counted in five random fields and the ratio of p-PERK staining cells was calculated. 
Data were presented as mean $\pm S D$. $N=5, \star \star \star \star$, means $p$-value $<0.001, N$.S means no significance. Con: control, I/R: ischemia/reperfusion, Cst: catestatin, Veh: vehicle.

A

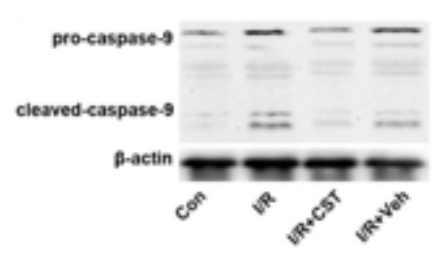

C

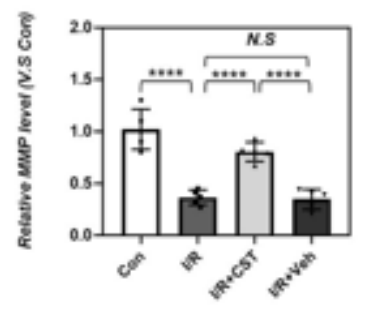

B

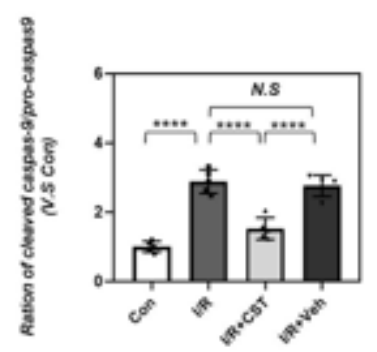

D

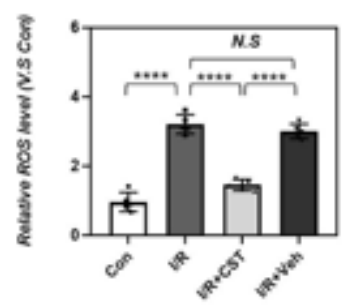

E

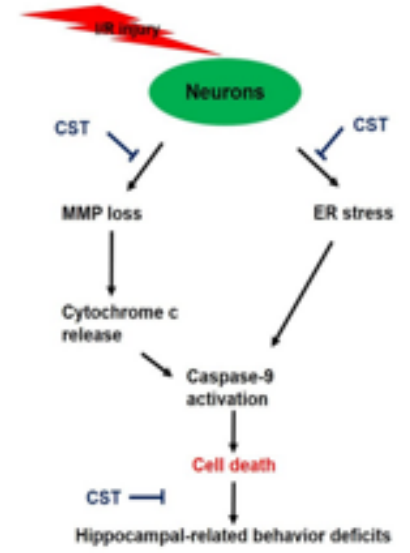

Figure 6

Effects of CST treatment on caspase-9 cleavage, MMP loss and ROS production in hippocampus neurons underwent $\mathrm{I} / \mathrm{R}$ injury $\mathrm{A}$. Western blot shows the caspase- 9 cleavage in each group, Con: control, I/R: ischemia/reperfusion, Cst: catestatin, Veh: vehicle. B. The quantification of cleaved caspase9/caspase-9 ration related panel A. Data was presented as mean $\pm S D$. $N=5, * \star \star$, means $p$-value $<0.001$, N.S means no significance. C. MMP level in each group, Con: control, l/R: ischemia/reperfusion, Cst: catestatin, Veh: vehicle. Data were presented as mean $\pm S D$. $N=5$, $\star \star \star \star$, means $p$-value $<0.001$, N.S means no significance. D. ROS production in each group, Con: control, I/R: ischemia/reperfusion, Cst: catestatin, Veh: vehicle. Data were presented as mean $\pm S D$. $N=5$, $\star \star \star \star$, means $p$-value $<0.001$, N.S means no significance. E. Pattern diagram of the mechanism by which CST exerts a protective effect on I/R injury. 